\title{
Determinación del punto de corte del porcentaje de PSA libre para la selección de pacientes sometidos a primera biopsia de próstata
}

\author{
A. López Luque, J. Gómez Bermudo, J. Márquez López, M. Leva Vallejo, J.C. Regueiro López, \\ $M^{a}=J$. Requena Tapia
}

Servicio de Urología. Hospital Regional Universitario Reina Sofia. Córdoba.

Actas Urol Esp 2006; 30 (1): 13-17

\section{RESUMEN}

DETERMINACIÓN DEL PUNTO DE CORTE DEL PORCENTAJE DE PSA LIBRE PARA LA SELECCIÓN DE PACIENTES SOMETIDOS A PRIMERA BIOPSIA DE PRÓSTATA

Objetivo: Determinar el punto de corte de \%PSA libre que nos permita optimizar la indicación de primera biopsia de próstata.

Material y métodos: Estudio prospectivo entre junio 2002 y septiembre 2004 en el que incluimos pacientes sometidos a primera biopsia de próstata con tacto rectal normal, niveles de PSA total entre 3-10 ng/ml y ecografia transrectal de próstata normal. Realizamos estudio estadístico descriptivo de las variables edad, PSA, volumen prostático y porcentaje de PSA libre. Realizamos análisis estadístico analítico mediante curvas ROC de las variables PSA y \% PSAL determinando cual de ellas predice mejor la existencia de cáncer de próstata en la primera biopsia transrectal de próstata.

Resultados: Se reclutan un total de 727 hombres con una edad media de 62,91 años, el PSA medio de 6,12 ng/ml, el volumen prostático medio $42,78 \mathrm{cc}$, el \% PSA libre medio de 15,22\%. Se diagnosticaron un total de 106 tumores de próstata, obteniendo una tasa de biopsia prostática del 14,6\%; 77 con afectación de 1 lóbulo y 29 con afectación de los 2 lóbulos. El gleason más frecuente fue el 6 (46 casos 43,4\%) seguido del gleason 7 (43 casos 40,6\%). En el análisis mediante curvas ROC el área bajo la curva para el PSA total fue de 0,476 (p=0,3) y para el \% PSA libre de 0,611 (p=0,023). El punto de corte óptimo para el \% PSA libre en nuestra serie se situó en el 19\% con una sensibilidad del 91,5\% y una especificidad del $20 \%$. El uso de este parámetro nos hubiese permitido la realización de 138 biopsias menos (19,11\%) con la pérdida de diagnóstico de 10 casos de cáncer de próstata.

Conclusiones: El uso del \%PSA libre es útil y permite en nuestro medio la mejor indicación de los pacientes que van a ser sometidos a primera biopsia de próstata, evitando la realización de biopsias innecesarias.

Palabras clave: Cáncer de próstata. PSA total. PSA libre. Curva ROC. Punto de corte. Biopsia transrectal ecodirigida prostática.

\section{ABSTRACT \\ DETERMINATION OF FREE PROSTATIC SPECIFIC ANTIGEN CUT POINT FOR THE SELECTION OF PATIENTS IN FIRST PROSTATE BIOPSY}

Objetives: Determine the cut point of free PSA rate for optimize the first prostate biopsy indication.

Material and methods: Prospective trial between june 2002-september 2004, We included patients in first prostate biopsy with normal rectal digital examen, total PSA between 3-10 ng/ml and normal transrectal prostate ultrasound. We realize descriptive stadistic analisis of variables age, total PSA, prostate volume and \% free PSA and analitic stadistic analisis with ROC curves of variables total PSA and \% free PSA for determine as of her predicts the best one rate of prostate cancer.

Results: We reclute 727 men with a mean age 62.91 years, total PSA mean $6.12 \mathrm{ng} / \mathrm{ml}$, prostate volume mean 42.78 cc and \% free PSA mean $15.22 \%$. We had 106 prostate cancer, the prostate cancer rate in first biopsy was $14.6 \% ; 77$ cases had 1 lobe prostate cancer and 29 in 2 lobes. The most frecuent gleason was 6 (46 cases $43.4 \%$ ) and the second gleason 7 (43 cases 40.6\%). In the ROC curves analysis, total PSA had area under the curve 0.476 ( $\mathrm{p}=0.3$ ) and 0.611 $(\mathrm{p}=0.023)$ for $\%$ free PSA. The optime cut point for $\%$ free PSA in our trial was 19\% (Sensibility $91.4 \%$ and Specificity $20 \%)$. The use of this cut point had allowed the saving us 138 biopsies (19.11\%) with the lost one of diagnose of 10 cases of cancer of prostate.

Conclusions: The use of the \% free PSA is useful and allows in our region the best indication of the patients who are going to first biopsy of prostate, avoiding the accomplishment of unnecessary biopsies.

Key words: Prostate cancer. Total PSA. Free PSA. ROC curve. Cut point. Tansrectal ultrasound prostate biopsy. 
$\mathrm{E}^{1}$ adenocarcinoma de próstata es el tumor maligno no derivado de la piel más frecuente en el hombre. Actualmente la técnica globalmente aceptada para el diagnóstico de cáncer de próstata es la biopsia transrectal ecodirigida (BTEP) y el estudio anatomopatológico de los cilindros obtenidos.

La indicación de la primera BTEP esta actualmente influenciada por variables clínicas y analíticas entre las que cabe destacar: la edad, el tacto rectal, en nivel de antígeno prostático específico (PSA), el porcentaje de PSA libre en la circulación sanguínea (\%PSAL) y la relación entre el volumen prostático y el PSA total también denominado densidad de PSA (dPSA).

La tasa de positividad para cáncer de próstata en pacientes con PSA entre 4 y $10 \mathrm{ng} / \mathrm{ml}$ y tacto rectal normal fue publicada entorno al $20-30 \%{ }^{1}$. Posteriormente surgieron valores de corte para el \%PSAL con la finalidad de optimizar la indicación de BTEP y mejorar la tasa de positividad de la misma, evitando la realización de biopsias innecesarias. Sin embargo, estos valores de corte han sido obtenidos de muestras de pacientes que proceden de poblaciones distintas a nuestro medio.

\section{OBJETIVOS}

El objetivo del estudio es la selección del punto de corte óptimo para el \%PSAL en un grupo de pacientes procedentes de nuestra región sanitaria.

\section{MATERIAL Y MÉTODOS}

Hemos realizado un estudio prospectivo que se inició en junio del 2002 y finalizó en septiembre del 2004 y en que se reclutaron pacientes que cumplían los siguientes criterios de inclusión:

- Varones sometidos a primera biopsia prostática.

- Tacto rectal determinado por al menos dos urólogos y definido como normal.

- PSA entre 3-10 ng/ml.

- Ecografia transrectal sin hallazgos sugestivos de cáncer de próstata.

A los pacientes que cumplían los criterios de inclusión en el estudio se les recogían las variables edad, tacto rectal, PSA total (determinación realizada con el reactivo Hybritech-Tandem-R), porcentaje de PSA libre (determinación realizada con el reactivo Hybritech prototipe free PSA.) y volumen prostático medido mediante ecografía transrectal.

Todos los pacientes fueron biopsiados por el mismo urólogo, y en todos ellos se tomaron al menos 6 muestras. Del informe anatomopatológico se recogian las variables: resultado de la biopsia y en caso de que hubiese un diagnóstico positivo para cáncer de próstata; el Gleason, afectación lobular, así como el porcentaje de cilindros afectados.

Una vez finalizado el periodo de recogida de datos se procesaron los mismos y se realizó un análisis estadístico descriptivo y un análisis de comparación de pruebas diagnósticas mediante curvas ROC, determinando el área bajo la curva para las variables PSA y \%PSAL. Los puntos de corte se establecieron con una sensibilidad y especificidad similar a los publicados en la literatura, el análisis estadístico se realizó mediante el programa bajo entorno Windows, SPSS v 11.0 (Chicago, Illinois).

\section{RESULTADOS}

Se obtuvieron un total de 727 pacientes. La Tabla 1 muestra las características de la serie global en la que la edad media fue de 62,91 años, el PSA medio de $6,12 \mathrm{ng} / \mathrm{ml}$, el volumen prostático medio $42,78 \mathrm{cc}$, el \% PSA libre medio de $15,22 \%$ y la densidad media de PSA de $0,17 \mathrm{ng} / \mathrm{ml} / \mathrm{cc}$.

En 229 casos se realizó biopsia sextante $(31,5 \%)$, en 94 casos se tomaron 8 muestras (12,9\%), en 194 casos se realizó biopsia con toma de 10 muestras $(26,7 \%)$ y en 210 casos se tomaron 12 muestras $(28,9 \%)$ obteniendo un total de 6.586 cilindros que fueron identificados en su totalidad como tejido prostático.

Se diagnosticó cáncer de próstata en 106 casos, lo cual supone una tasa de primera biopsia prostática positiva del $14,6 \%$; las Tablas 2 y 3 muestran la distribución de los casos en función del resultado anatomopatológico y del Gleason así como el número de cilindros positivos respectivamente.

La comparación de predictividad realizada mediante el análisis con curvas ROC (Fig. 1) de las variables \%PSAL y PSA total pone de manifiesto que la variable \%PSAL presenta una mejor predictividad de cáncer de próstata (área bajo la curva 0,611 vs 0,476 ). 
Tabla 1

Características de la serie

\begin{tabular}{lccccc}
\hline & \multicolumn{4}{c}{ Caracteristicas de la muestra } & \\
& Edad & PSA & $\begin{array}{c}\text { Volumen } \\
\text { Prostático }\end{array}$ & \%PSA-libre & Densidad \\
& 727 & 727 & 727 & 727 & PSA \\
\hline N & 62,91 & 6,1213 & 41,7859 & 15,2213 & 0,1710 \\
Media & 9,10 & 1,7613 & 17,7915 & 5,5898 & 0,0800 \\
Desv. Tip. & 9 & & & & \\
\hline
\end{tabular}

Tabla 2

Resultado anatomopatológico de las biopsias obtenidas

\begin{tabular}{lcccc}
\hline \multicolumn{4}{c}{ Resultado de biopsia } & \\
& Frecuencia & Porcentaje & $\begin{array}{c}\text { Porcentaje } \\
\text { válido }\end{array}$ & $\begin{array}{c}\text { Porcentaje } \\
\text { acumulado }\end{array}$ \\
\hline 1 lóbulo & 72 & 9,9 & 9,9 & 9,9 \\
1 lóbulo +pinag & 5 & 0,7 & 0,7 & 10,6 \\
2 lóbulos & 29 & 4,0 & 4,0 & 14,6 \\
HBP & 475 & 65,3 & 65,3 & 79,9 \\
Pinag & 17 & 2,3 & 2,3 & 82,3 \\
P. inflamatorio & 129 & 17,7 & 17,7 & 100,0 \\
Total & 727 & 100,0 & 100,0 & \\
\hline
\end{tabular}

Solamente la variable \%PSAL presenta una área bajo la curva estadísticamente significativa $(\mathrm{p}=0,023)$.

La Tabla 4 pone de manifiesto los distintos puntos de corte de \%PSAL en función de una sensibilidad y especificidad determinada; de tal manera que asumiendo unos valores de las mismas similares a los publicados en la literatura, en nuestra serie, el punto de corte se situaría entorno al $19 \%$.

\section{Tabla 3}

Distribución del cáncer de próstata en función del Gleason

\section{Gleason}

\begin{tabular}{cccc} 
& & Frecuencia & Porcentaje \\
\hline Gleason & 4 & 1 & 0,1 \\
6 & 46 & 43,4 \\
7 & 43 & 40,6 \\
8 & 11 & 10,4 \\
9 & 5 & 4,7 \\
& 106 & 14,6 \\
\hline
\end{tabular}

La Tabla 5 define en nuestra serie el número de tumores prostáticos que se diagnosticarían, el de los que quedarían sin diagnosticar y el número de biopsias ahorradas.

\section{DISCUSIÓN}

$\mathrm{El}$ antígeno prostático específico (PSA) es el marcador tumoral más importante y específico de todos los descritos en la oncología humana. Sin embargo, a pesar de ello su especificidad es baja, lo que implica un importante número de falsos positivos que determinan la realización de biopsia transrectales ecodirigidas prostáticas (BTEP) innecesarias.

La BTEP es una técnica invasiva que no está exenta de complicaciones, en la literatura $^{2}$ encontramos tasas de sangrado rectal del 2,1-2,4\%, hematuria moderada-severa de $0,5-1 \%$, síncopes vasovagales $(1,4-2-8 \%)$, ITU leves-moderadas (10,9-15\%), sepsis urinarias $(0,1-0,3 \%)$, sangrado rectal o hematuria que requiere intervención quirúrgica $(0,1 \%)$ y muerte $(0,05 \%)$. Asímismo, el riesgo de complicaciones aumenta proporcionalmente con las siguientes rebiopsias ${ }^{3}$.

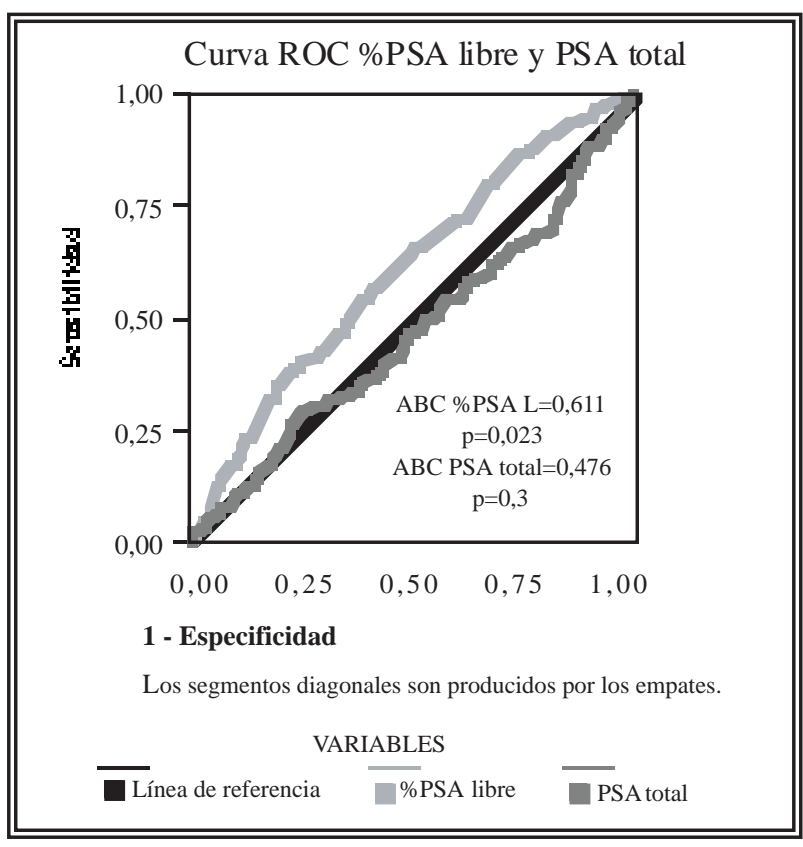

FIGURA 1. Comparación de curvas Roc para las variables \%PSA libre y PSA total. 
Tabla 4

Sensibilidades y especificidades en función del punto de corte para el \%PSA libre

\begin{tabular}{ccc}
\hline $\begin{array}{c}\text { Puntos de corte } \\
\text { \% PSA libre }\end{array}$ & Sensibilidad & Especificidad \\
\hline $10 \%$ & $\mathbf{3 2 , 1} \%$ & $81,8 \%$ \\
$15 \%$ & $65,1 \%$ & $51,1 \%$ \\
$17 \%$ & $80,2 \%$ & $33,5 \%$ \\
$18 \%$ & $86 \%$ & $26,1 \%$ \\
$19 \%$ & $\mathbf{9 1 , 5} \%$ & $\mathbf{2 0} \%$ \\
$20 \%$ & $94,3 \%$ & $14,3 \%$ \\
$21 \%$ & $95,3 \%$ & $11,9 \%$ \\
$25 \%$ & $99,1 \%$ & $6,3 \%$ \\
$27,5 \%$ & 100 & $3,5 \%$ \\
\hline
\end{tabular}

\section{Tabla 5}

Rentabilidad obtenida por los distintos puntos de corte y cánceres de próstata sin diagnosticar en función de ellos

\begin{tabular}{cccc}
\hline $\begin{array}{c}\text { Punto de } \\
\text { corte \% } \\
\text { PSA libre }\end{array}$ & $\begin{array}{c}\text { Casos } \\
\text { de CP } \\
\text { diagnosticados }\end{array}$ & $\begin{array}{c}\text { Casos } \\
\text { de CP sin } \\
\text { diagnosticar }\end{array}$ & $\begin{array}{c}\text { Biopsias } \\
\text { horradas }\end{array}$ \\
\hline $15 \%$ & 69 & 37 & 349 \\
$17 \%$ & 84 & 22 & 230 \\
$18 \%$ & 91 & 15 & 177 \\
$19 \%$ & 96 & 10 & 138 \\
$20 \%$ & 99 & 7 & 96 \\
$21 \%$ & 100 & 6 & 80 \\
$22 \%$ & 100 & 6 & 72 \\
$23 \%$ & 100 & 6 & 68 \\
$24 \%$ & 103 & 3 & 49 \\
$25 \%$ & 104 & 2 & 30 \\
\hline
\end{tabular}

Esta razón, asociada a la escasa rentabilidad diagnóstica de la biopsia explica la utilidad de variables clínicas y analíticas que nos permiten seleccionar al paciente que va a ser sometido a BTEP con la finalidad de optimizar el diagnóstico de cáncer de próstata, evitando la realización de biopsias, las complicaciones y el estrés que supone para el paciente la realización de la misma.

Muchos han sido los autores que han promulgado el uso del \%PSAL, definido como la tasa en sangre periférica entre el PSA libre (no unido a proteínas) y el PSA total.
Lilja et al. ${ }^{4}$ fueron los primeros en desarrollar en 1991 el método bioquímico por el cual se discriminaba los niveles en sangre de la forma libre del PSA frente al PSA total. En 1995 Luderer et al. ${ }^{5}$ promulgaron por primera vez el uso del porcentaje de PSA libre (\%PSAL) para la indicación de biopsia de próstata en la zona gris de PSA total (PSA 4-10 ng/ml). En su estudio mediante curvas ROC demostraron que aisladamente el PSA total entre 4-10 ng/ml no era capaz de determinar con precisión la positividad o no de cáncer de próstata en la BTEP y en contraste, el \%PSAL era útil para identificar aquellos hombres con cáncer. Ellos observaron una especificidad del 31\% para el \%PSAL frente a un 0\% del PSA total y establecieron el punto de corte en un $25 \%$. Los resultados de nuestro estudio confirman las conclusiones de Luderer, ya que en nuestra serie la curva ROC obtenida para el PSA total no nos permite afirmar con una significación estadística adecuada que sea un buen predictor de positividad para cáncer de próstata en los pacientes seleccionados.

Muchos han sido los estudios posteriores que hay publicados en la literatura presentando puntos de corte que oscilan entre el $14 \%$ y el $26 \%$. Catalona et al. ${ }^{6}$ publican en 1998 un estudio prospectivo multicéntrico en el que se establece inicialmente el punto de corte en un $25 \%$ con una sensibilidad del $90 \%$ y especificidad del 20\% para pacientes con PSA entre 4-10 ng/ml y llegando a la conclusión que usando este punto de corte se ahorrarian aproximadamente un $20 \%$ de las biopsias realizadas. Posteriormente este mismo autor incluye el subgrupo de pacientes con PSA entre 2,6-4 $\mathrm{ng} / \mathrm{ml}$, estableciendo, con una sensibilidad del 90\% el punto de corte en $26 \%$ para este subgrupo.

En nuestro estudio, el punto de corte obtenido para una sensibilidad del $90 \%$ y una especificidad del $20 \%$ es del $19 \%$. Si hubiésemos incluido este valor como criterio de inclusión, habríamos ahorrado la realización de 139 biopsias $(19,11 \%)$ y la tasa de biopsia prostática positiva hubiese ascendido del 14,6\% al 18,02\%, dejando sin diagnosticar 10 tumores prostáticos.

En nuestro medio, hasta la publicación de este estudio, el punto de corte del \%PSAL que utilizábamos para la indicación de primera biopsia era del 20\%; el bajarlo al 19\% en nuestra serie nos hubiese permitido ahorrar 42 biopsias diagnosticando 3 tumores menos. 
El punto de corte del \%PSAL es un valor que depende de factores como la incidencia de cáncer de próstata en la población, la técnica de la biopsia de próstata, la edad del paciente, el nivel de PSA, el volumen prostático, el método bioquímico utilizado para la determinación tanto del PSA total como de su forma libre e incluso de la raza del paciente. En un estudio publicado por Fowler et al. ${ }^{7}$ en el 2000 se llega a la conclusión que una proporción importante de hombres afro-americanos con un \%PSAL superior al 25\% presentaban una mayor probabilidad de presentar cáncer de próstata en contraste con hombres caucasianos (32 vs. 13\%).

Asimismo, este punto de corte no se define por ningún método estadístico, sino que debe ser el clinico quien determine que valor de sensibilidad (S) y especificidad (E) es razonable para conseguir un equilibrio aceptable entre el número de biopsias ahorradas y el número de varones que quedan sin diagnosticar.

En nuestra serie, para tener un nivel de sensibilidad del 100\% deberíamos establecer el punto de corte en el 27,5\%, con el que hubiésemos diagnosticado todos los tumores y hubiéramos ahorrado 25 biopsias (3,5\%). Hemos tomado el punto de corte en el 19\% porque presenta un nivel de S y E similar al publicado por otros autores en la literatura.

La novedad que, pensamos, presenta nuestro estudio es que se trata de un estudio prospectivo, reciente y realizado de forma homogénea a una muestra que procede de una población que es más representativa de la población española que el resto de los estudios publicados en la literatura, y que por tanto podría ser útil para la práctica clinica en nuestra región.
Aún así, sería necesario la publicación de otros estudios similares realizados en otras regiones españolas, y aunque estos estudios sirviesen de referencia pensamos que el nivel de corte para el $\%$ PSAL debería ser establecido en cada centro en función de sus resultados, ya que podría verse influenciado por los factores comentados anteriormente.

\section{REFERENCIAS}

1. Hodge KK, McNeal JE, Terris MK, Stamey TA. Ramdom systematic versus directed ultrasound guided transrectal core biopsies of the prostate J Urol 1989;142(1):71-74; discussion 74-75.

2. Roberts RO, Bergtralh EK, Besse JA, Lieber MM, Jacobsen SJ. Trends and risk factors for prostate biopsy complications in the pre-PSA and PSA eras, 1980-1997. Urology 2002;59(1):79-84.

3. Djavan B, Zlotta AR, Marberger M, Schulman CC. Complications of TRUS biopsy. Revision. J Urol 156:856

4. H. Lilja A, Christensson U, Dahlen MT, Matikainen O, Nilsson K. Pettersson T. Lövgren. Prostate-Specific antigen in human serum occurs predominately in complex with a1antichymotrypsin. Clin Chem 1991;37:1618-1625.

5. Luderer AA, Chen YT, Soriano TF, Kramp WJ, Carlson G, Cuny C, et al. Measurement of the proportion of free to total PSA improves diagnostic performance of PSA in the diagnostic gray zone of total PSA. Urology 1995;46:187-194.

6. Catalona WJ, Partin AW, Slawin KM, Brawer MK, Flanigan RC, Patel A, et al. Use of the percentage of free prostate-specific antigen to enhance differentiation of prostate cancer from benign prostatic disease: A prospective multicenter clinical trial. JAMA 1998;279:1542-1547.

7. Fowler JE Jr, Sanders J, Bigler SA, Rigson J, Kilambi NK, SA. L. Percent free prostate specific antigen and cancer detection in black and white men with total specific antigen 2.4 to $9.9 \mathrm{ng} / \mathrm{ml}$. J Urol 2000;163:1467-1470.

Dr. A. López Luaue

E-mail: allo2198@supercable.es

(Trabajo recibido el 3 de junio 2005) 